\title{
ON A MASS DISTRIBUTION ASSOCIATED WITH A CLASS OF POLYNOMIALS
}

\author{
MAXWELL O. READE ${ }^{1}$
}

1. Let $\mathcal{D}$ denote a bounded, simply connected domain in the $x, y$-plane, and let $E$ be a closed set lying in $\mathscr{D}$. If $\lambda(e) \not \equiv 0$ is a finite, completely additive distribution of mass (of arbitrary sign) defined for Borel sets $e$ in $E$, then the couple $(E, \lambda)$ will be called a distribution (completely interior to $\mathscr{D})$. If $\left(E_{1}, \lambda_{1}\right)$ is another distribution, completely interior to $\mathscr{D}$, such that $E$ and $E_{1}$ are similar and such that homologous parts of $E$ and $E_{1}$ have the same mass, then $(E, \lambda)$ and $\left(E_{1}, \lambda_{1}\right)$ are said to be similar distributions. The distribution $(E, \lambda)$ is said to be normal if and only if all its harmonic moments vanish, that is, if and only if

$$
\begin{aligned}
\iint_{E} \rho^{n} \cos n \theta d \lambda=0, \quad \iint_{E} \rho^{n} \sin n \theta d \lambda & =0, \\
n & =0,1,2, \ldots .
\end{aligned}
$$

Now let $f(x, y)$ be a real function, continuous in $\mathscr{D}$, and let $\left(E_{0}, \lambda_{0}\right)$ be a distribution that is completely interior to $\mathscr{D}$. Then $f(x, y)$ and $\left(E_{0}, \lambda_{0}\right)$ are said to be associated if, and only if,

$$
\iint_{E} f(x, y) d \lambda=0
$$

holds for each distribution $(E, \lambda)$ that is both similar to $\left(E_{0}, \lambda_{0}\right)$ and completely interior to $\mathcal{D}$.

The interesting ideas contained in the preceding paragraphs are due to Choquet and Deny [2] ${ }^{2}$ who determined classes of continuous functions associated with distributions. One of their results is the following one.

THEOREM A [2, p. 131]. If $f(x, y)$ is real and continuous in $\mathcal{D}$, if $(E, \lambda)$ is a non-normal distribution completely interior to $\mathcal{D}$, with $p$ the order of the first nonvanishing harmonic moment, and if $f(x, y)$ and

Received by the editors June 6, 1949 and, in revised form, November 1, 1949.

${ }^{1}$ The author gratefully acknowledges financial aid given by the Engineering Research Institute, University of Michigan, under Project 7865 contract N8-ONR-581.

2 Numbers in brackets refer to the bibliography at the end of the paper. 
$(E, \lambda)$ are associated, then $f(x, y)$ satisfies the differential equations

$$
\begin{aligned}
& \lambda^{p} f(x, y) \equiv\left(\frac{\partial}{\partial x}+i \frac{\partial}{\partial y}\right)^{p} f(x, y)=0, \\
& \bar{\lambda}^{p} f(x, y) \equiv\left(\frac{\partial}{\partial x}-i \frac{\partial}{\partial y}\right)^{p} f(x, y)=0
\end{aligned}
$$

throughout $\mathscr{D}$.

As Choquet and Deny remark, it follows from (3) that $f(x, y)$ is a polynomial of the form

$$
f(x, y)=\sum_{s=0}^{p-2}\left(x^{2}+y^{2}\right)^{p-s-1} \psi_{s}(x, y)+\Psi_{p-1}(x, y),
$$

where $\psi_{s}(x, y)$ is a homogeneous polynomial of degree $s, s=0,1,2$, $\cdots, p-2$, and where $\Psi_{p-1}(x, y)$ is an arbitrary polynomial of degree at most $p-1$.

In this note we shall make use of Theorem A to obtain characterization theorems for polynomial solutions of the equation

$$
\Delta^{k} f(x, y)=0,
$$

where $k$ is an integer, $k \geqq 1$. These new results are generalizations of earlier results due to Beckenbach and Reade [1], Haskell [3], and Poritsky [5].

2. We shall use mean-value functions of the form

$$
\begin{aligned}
& L(f ; x, y ; r, \phi) \equiv \frac{1}{\left\|p_{n}\right\|} \int_{p_{n}(x, y ; r, \phi)} f(\xi, \eta) d s, \\
& A(f ; x, y ; r, \phi) \equiv \frac{1}{\left\|P_{n}\right\|} \iint_{P_{n}(x, y ; r, \phi)} f(\xi, \eta) d \xi d \eta .
\end{aligned}
$$

Here $P_{n}(x, y ; r, \phi)$ is the finite, closed region bounded by the regular $n$-gon $p_{n}(x, y ; r, \phi)$ with center $(x, y)$, inscribed circle of radius $r$, "orientation" $\phi,-\pi / n<\phi \leqq \pi / n$. $\left\|p_{n}\right\|=2 n r \tan (\pi / n)$ is the length of $p_{n}(x, y ; r, \phi)$, and $\left\|P_{n}\right\|=n r^{2} \tan (\pi / n)$ is the area of $P_{n}(x, y ; r, \phi)$.

It is well known that the areal mean-value $A(f ; x, y ; r, \phi)$ is one degree smoother than $f(x, y)$; that is, if $f(x, y)$ is superficially summable over $\mathcal{D}$, then the areal mean-values are continuous where they are defined, and if $f(x, y)$ is of class $C^{p}$ in $\mathcal{D}$, then the meanvalue functions are of class $C^{p+1}$ where defined.

We shall also make use of the following easily established identities. If $l, m, n$ are positive integers, then 
(6)

$$
\begin{gathered}
\sum_{m=1}^{n} e^{i(\phi+(2 \pi l / n) m)}=n \delta_{l, n} e^{i \phi}, \\
\int_{p_{n}(0,0 ; r, \phi)} \rho^{l} e^{i l \theta} d s=n \delta_{l, n} r^{l+1} \int_{-\pi / n}^{\pi / n} e^{i l(\theta+\phi)} \sec ^{l+2} \theta d \theta,
\end{gathered}
$$

where $\delta_{l, n}=1$ if $l$ is an integral multiple of $n$, and $\delta_{l, n}=0$ otherwise.

A set of $k$ concentric and similarly oriented regular $n$-gons $p_{n}\left(x, y ; r_{1}, \phi\right), p_{n}\left(x, y ; r_{2}, \phi\right), \cdots, p_{n}\left(x, y ; l_{n}, \phi\right)$ will be called a $k$-tuple of polygons, and will be denoted by both $\left\{p_{n}\left(x, y ; r_{k}, \phi\right)\right\}$ and $\left\{P_{n}\left(x, y ; r_{k}, \phi\right)\right\}$.

If $\left\{p_{n}\left(x, y ; r_{k}, \phi\right)\right\}$ is a $k$-tuple lying in $\mathscr{D}$, and if $f(x, y)$ is summable on each $p_{n}\left(x, y ; r_{s}, \phi\right), s=1,2, \cdots, k$, then the symbol

$$
\operatorname{det}\left[L\left(f ; x, y ; \boldsymbol{r}_{k}, \boldsymbol{\phi}\right)-f(x, y) \boldsymbol{r}_{k}^{2} \boldsymbol{r}_{k}^{4} \cdots \boldsymbol{r}_{k}^{2 k-2}\right]
$$

will denote the determinant whose $k$ th row appears within the brackets. A similar definition is attached to the symbol

$$
\operatorname{det}\left[A\left(f ; x, y ; \boldsymbol{r}_{k}, \boldsymbol{\phi}\right)-f(x, y) \underset{\boldsymbol{r}_{k} \boldsymbol{r}_{k}^{4}}{2} \cdots \boldsymbol{r}_{k}^{2 k-2}\right] .
$$

Unless explicitly stated otherwise, $n$ and $k$ will always denote fixed integers, $n \geqq 3, k \geqq 1$.

3. The following result is fundamental for this paper.

Lemma 1. If $f(x, y)$ is superficially summable over $\mathcal{D}$, if $f(x, y)$ is summable over each $p_{n}(x, y ; r, \phi)$ lying in $\mathcal{D}$, and if

$$
\operatorname{det}\left[L\left(f ; x, y ; r_{k}, \phi\right)-f(x, y) r_{k}^{2} r_{k}^{4} \cdots r_{k}^{2 k-2}\right]=0
$$

holds for each $k$-tuple $\left\{p_{n}\left(x, y ; r_{k}, \phi\right)\right\}$ lying in $\mathcal{D}$, then $f(x, y)$ is a polynomial of degree at most $2 p-2$, satisfying (3); here $p=n$, if $n$ is odd, and $p=\delta n$, the smallest integral multiple of $n$ for which $\delta n>2 k-2$, when $n$ is even.

Proof. Under the present hypotheses, if we multiply the $t$ th row of the left-hand member of (7) by $2 n r_{t} \tan (\pi / n)$, then integrate with respect to $r_{t}$, and then divide by $n r_{t}^{2} \tan (\pi / n)$, for $t=0,1, \cdots, k$, then we obtain

$$
\operatorname{det}\left[A\left(f ; x, y ; r_{k}, \phi\right)-f(x, y) r_{k}^{2} r_{k}^{4} \cdots r_{k}^{2 k-2}\right]=0,
$$

which must hold for all $k$-tuples $\left\{p_{n}\left(x, y ; r_{k}, \phi\right)\right\}$ lying in $\mathscr{D}$. If we expand $(8)$, then we find $f(x, y)$ expressed as a linear combination of 
areal mean-value functions. The latter are one degree smoother than $f(x, y)$, where defined. Hence it follows by an induction that $f(x, y)$ has derivatives of all orders in $\mathcal{D}$.

To show that $f(x, y)$ is a polynomial satisfying an equation of the form (3), we shall exhibit a non-normal distribution $\left(E_{0}, \lambda_{0}\right)$, completely interior to $\mathcal{D}$ and associated with $f(x, y)$. Then we shall appeal to Theorem A. Without loss of generality, we may assume that the origin is in $\mathcal{D}$. Let $\left\{p_{n}\left(0,0 ; \rho_{k}, 0\right)\right\}$ denote a fixed $k$-tuple of distinct $n$-gons lying in $\mathcal{D}$. Then ( 7$)$ holds for this $k$-tuple:

$$
\operatorname{det}\left[L\left(f ; 0,0 ; \rho_{k}, 0\right)-f(0,0) \underset{\rho_{k}^{2} \rho_{k}^{4}}{2} \cdots \rho_{k}^{2 k-2}\right]=0 .
$$

If we choose real constants $m_{t}$ proportional to the cofactors of the $L\left(f ; 0,0 ; \rho_{t}, 0\right)-f(0,0)$ in $(9)$ such that

$$
\sum_{t=1}^{k} m_{t}=: 1
$$

then

$$
\sum_{t=1}^{k} m_{t}\left[L\left(f ; 0,0 ; \rho_{t}, 0\right)-f(0,0)\right]=0
$$

must hold. The distribution we shall use is now defined to consist of a mass $m_{t}$ distributed uniformly on $p_{n}\left(0,0 ; \rho_{t}, 0\right), t=1,2, \cdots, k$, and of a mass $(-1)$ at the origin, while the corresponding set $E_{0}$ is defined to be the smallest convex set containing the $k$-tuple. From (10) and (11) it follows that the total mass is zero and

$$
\iint_{E_{0}} f(x, y) d \lambda_{0}=\sum_{t=1}^{k} m_{t}\left[L\left(f ; 0,0 ; \rho_{t}, 0\right)-f(0,0)\right]=0 .
$$

It follows from (7) and the way in which the constants $m_{t}$ were defined that (2) holds for each distribution $(E, \lambda)$ which is similar to $\left(E_{0}, \lambda_{0}\right)$ and which lies completely interior to $D$, that is, (2) holds for each distribution $(E, \lambda)$ obtained by distributing the mass $m_{t}$ uniformly over $p_{n}\left(x, y ; r_{t}, \rho\right)$, for each member of a $k$-tuple $\left\{p_{n}\left(x, y ; r_{k}, \phi\right)\right\}$ that is similar to the $k$-tuple $\left\{p_{n}\left(0,0 ; \rho_{k}, 0\right)\right\}$ and that lies in $\mathscr{D}$. Hence $\left(E_{0}, \lambda_{0}\right)$ is associated with $f(x, y)$.

We shall now show that the distribution $\left(E_{0}, \lambda_{0}\right)$ defined above is not a normal one. To that end, we examine

$$
\iint_{E_{0}} \rho^{l} e^{i l \theta} d \lambda_{0}=\operatorname{det}\left[L\left(\rho^{l} e^{i l \theta} ; 0,0 ; \rho_{k}, 0\right) \rho_{k}^{2} \rho_{k}^{4} \cdots \rho_{k}^{2 k-2}\right]
$$


which, by (6), becomes

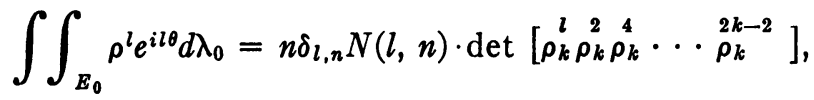

where

$$
N(l, n) \equiv K(l, n)+i M(l, n) \equiv \int_{-\pi / n}^{\pi / n} e^{i l \theta} \sec ^{l+2} \theta d \theta .
$$

We distinguish two cases. (a) $n$ odd. It follows from the nature of the determinant in (13), and from (14), that $l=n$ is the order of the first nonvanishing harmonic moment of $\left(E_{0}, \lambda_{0}\right)$. Therefore, by Theorem A, $f(x, y)$ satisfies (3) with $p=n$. (b) $n$ even. It follows from (13) and (14) that the first nonvanishing harmonic moment of $\left(E_{0}, \lambda_{0}\right)$ is that whose order is the first multiple of $n$ that exceeds $2 k-2$, say $\delta n$. Hence $f(x, y)$ satisfies (3) with $p=\delta n$. This completes the proof.

COROLlaRY 1. The conclusion of Lemma 1 is valid if (7) is replaced by (8) in the hypothesis.

Proof. We merely retrace the steps that led from (7) to (8) in the proof above.

We remark here that the order of the differential equation (3), valid for the conclusion of Lemma 1 , is not necessarily minimal; the order is a function of the first nonvanishing harmonic moment of distributions associated with $f(x, y)$. It will follow from our later results that if we assume that $k$ is the smallest integer that will work in (7), then the order $p$ obtained in the lemma is actually minimal.

The following observation will be useful. If (7) holds for each $k$ tuple $\left\{p_{n}\left(x, y ; r_{k}, \phi\right)\right\}$ lying in $\mathcal{D}$, then it follows that for each fixed point $(x, y)$ in $\mathcal{D}$ and for each fixed angle $\phi,-\pi / n<\phi \leqq \pi / n$, the mean-value functions $L(f ; x, y ; r, \phi), A(f ; x, y ; r, \phi)$ are polynomials in $r^{2}$ of degree at most $k-1$; that is, there exist functions $L_{s}(x, y ; \phi)$, $A_{8}(x, y ; \phi)$, such that

$$
\begin{aligned}
& L(f ; x, y ; r, \phi)=f(x, y)+\sum_{s=1}^{k-1} r^{2 s} L_{s}(x, y ; \phi), \\
& A(f ; x, y ; r, \phi)=f(x, y)+\sum_{s=1}^{k-1} r^{2 s} A_{s}(x, y ; \phi) .
\end{aligned}
$$

If $f(x, y)$ satisfies (4) in $D$, then we say that $f(x, y)$ is harmonic of order $k$ in $\mathscr{D}$; if $k=1$, we say that $f(x, y)$ is harmonic in $\mathcal{D}$. If $f(x, y)$ is harmonic of order $k$ in $\mathcal{D}$, and if $\left(x_{0}, y_{0}\right)$ is an arbitrary fixed point 
in $\mathcal{D}$, then it is known that there exist functions $G_{s}(x, y)$, harmonic in $\mathcal{D}$, such that $[4$, p. 13$]$

$$
f(x, y)=\sum_{s=0}^{k-1}\left[\left(x-x_{0}\right)^{2}+\left(y-y_{0}\right)^{2}\right] G_{s}(x, y)
$$

holds within the largest star-like domain with "center" $\left(x_{0}, y_{0}\right)$ lying in $\mathscr{D}$. If we expand each $G_{s}(x, y)$ in a Fourier series about $\left(x_{0}, y_{0}\right)$, then we obtain

$$
\begin{aligned}
f(x, y) & =f\left(x_{0}+\rho \cos \theta, y_{0}+\rho \sin \theta\right) \\
& =\sum_{s=0}^{k-1} \rho^{2 s} \sum_{t=0}^{\infty} \rho^{t}\left(a_{s, t} \cos t \theta+b_{s, t} \sin t \theta\right) .
\end{aligned}
$$

This latter result leads to the following lemma.

LemMa 2. If $f(x, y)$ is superficially summable over $\mathcal{D}$, if $f(x, y)$ is summable over each $p_{n}(x, y ; r, \phi)$ lying in $\mathcal{D}$, and if (7) (or (8)) holds for each $k$-tuple $\left\{p_{n}\left(x, y ; r_{k}, \phi\right)\right\}$ lying in $\mathcal{D}$, then $f(x, y)$ is harmonic of order $k$ in $D$.

Proof. First suppose that $n$ is even. Under the present hypothesis, it follows from Lemma 1 that $f(x, y)$ is a polynomial of degree at most $2 \delta n-2$, satisfying

$$
\lambda^{\delta n} f(x, y)=0, \quad \bar{\lambda}^{\delta n} f(x, y)=0
$$

throughout $\mathcal{D}$. It follows from (18) that $\Delta^{\delta n} f(x, y)=0$ throughout $\mathcal{D}$, so that $f(x, y)$ is harmonic of order $\delta n$ in $\mathcal{D}$.

Now let $\left(x_{0}, y_{0}\right)$ be an arbitrary fixed point in $\mathcal{D}$. Then there exist functions $G_{1}(x, y), \cdots, G_{\delta n-1}(x, y)$, harmonic in $\mathcal{D}$, whose Fourier expansions about $\left(x_{0}, y_{0}\right)$ lead to the following analogue of (17):

$$
f(x, y)=\sum_{s=0}^{\delta n-1} \sum_{t=0}^{\infty} \rho^{2 s+t}\left(a_{s, t} \cos t \theta+b_{s, t} \sin t \theta\right),
$$

which must hold in a neighborhood of $\left(x_{0}, y_{0}\right)$. Then a direct computation in which we make use of (6) and (14) yields

$$
\begin{aligned}
& L\left(f ; x_{0}, y_{0} ; r, \phi\right) \\
& \quad=\frac{n}{\tan (\pi / n)} \sum_{s=0}^{\delta n-1} \sum_{t=0}^{\infty} r^{2 s+n t} K(n t, n)\left(a_{s, n t} \cos n t \phi+b_{s, n t} \sin n t \phi\right) .
\end{aligned}
$$

It now follows from (15) and the present hypotheses that the coefficient of $r^{2 k}$ in (20) must vanish:

$$
K(0, n) a_{k, 0}+\{\cos n t \phi, \sin n t \phi\}=0,
$$


where the braces indicate a linear expression in $\cos n \phi, \sin n \phi$, $\cos 2 n \phi, \sin 2 n \phi, \cdots$. But $K(0, n) \neq 0$ and (21) holds for all $\phi,-\pi / n$ $<\phi \leqq \pi / n$. Hence it follows by the usual Fourier analysis that (21) implies $a_{k, 0}=0$. But it is easily verified that

$$
a_{k, 0}=G_{k}\left(x_{0}, y_{0}\right)=\frac{1}{2^{2 k}} \frac{1}{k !} \frac{1}{(k+1) !} \Delta^{k} f\left(x_{0}, y_{0}\right) .
$$

Hence $\Delta^{k} f\left(x_{0}, y_{0}\right)=0$ at the arbitrary point $\left(x_{0}, y_{0}\right)$ in $\mathcal{D}$, and therefore throughout $\mathcal{D}$. Hence $f(x, y)$ is harmonic of order $k$ in $\mathscr{D}$.

If $n$ is an odd integer, then a similar analysis would yield the other part of the lemma.

THEOREM 1. If $f(x, y)$ is superficially summable over $\mathcal{D}$, and if $f(x, y)$ is summable over each $p_{n}(x, y ; r, \phi)$ in $\mathcal{D}$, then a necessary and sufficient condition that (7) hold for each $k$-tuple $\left\{p_{n}\left(x, y ; r_{k}, \phi\right)\right\}$ lying in $\mathcal{D}$ is that $f(x, y)$ be a polynomial, harmonic of order $k$, having one of the following representations.

(a) $n$ even:

$$
f(x, y)=\sum_{s=1}^{k}\left(x^{2}+y^{2}\right)^{\bullet-1} H_{s, \delta n-s}(x, y) .
$$

(b) $n$ odd:

$$
f(x, y)=\sum_{s=1}^{\min (k, n)}\left(x^{2}+y^{2}\right)^{-1} H_{s, n-s}(x, y) .
$$

Here $H_{s, j}(x, y)$ denotes a harmonic polynomial of degree at most $j$.

Necessity. First suppose that $n$ is even. It follows from Lemma 2 that $f(x, y)$ can be represented in the form (16), with $p=\delta n$, about the arbitrary, fixed point $\left(x_{0}, y_{0}\right)$ in $\mathcal{D}$. If we write

$$
\left.G_{s}(x, y)=\sum_{t=0}^{\infty}\left[\alpha_{s, t}\left(z-z_{0}\right)^{t}+\bar{\alpha}_{s, t} \overline{\left(z-z_{0}\right.}\right)^{t}\right], \quad 0 \leqq s \leqq k-1,
$$

where $z-z_{0} \equiv\left(x-x_{0}\right)+i\left(y-y_{0}\right)$, and where the bar denotes the complex conjugate, then (16) and Lemma 2 yield

$$
\begin{aligned}
f(x, y)= & \sum_{p=0}^{k-1}\left(z-z_{0}\right)^{p}\left\{\sum_{s=0}^{p} \alpha_{s, p-s} \overline{\left(z-z_{0}\right)^{p-s}}+\sum_{t=0}^{\infty} \bar{\alpha}_{p, t} \overline{\left(z-z_{0}\right)^{p+t}}\right\} \\
& +\sum_{p=k}^{\infty}\left(z-z_{0}\right)^{p} \sum_{s=0}^{k-1} \alpha_{s, p \rightarrow s} \overline{\left(z-z_{0}\right)^{p-s}} .
\end{aligned}
$$


Now by Lemma $1, f(x, y)$ satisfies (18) in $D$. Hence it follows that the coefficient of $z^{p}$ in (25) must vanish identically for all $p \geqq \delta n$; that is,

$$
\sum_{s=0}^{k-1} \alpha_{s, p-s}{\overline{\left(z-z_{0}\right)}}^{p-s} \equiv 0, \quad p \geqq \delta n
$$

Since (26) holds in a disc about $z_{0}$, it follows that

$$
\alpha_{s, p-s}=0, \quad 0 \leqq s \leqq k-1, p \geqq \delta n,
$$

must hold.

From (23) and (25) and (27) it follows that $f(x, y)$ has the representation

$$
\begin{aligned}
f(x, y)=\sum_{s=0}^{k-1}\left(z-z_{0}\right)^{s} \overline{\left(z-z_{0}\right)^{s}} \sum_{t=0}^{\delta n-s-1}\left[\alpha_{s, t}\left(z-z_{0}\right)^{t}\right. & \\
& \left.+\bar{\alpha}_{8, t}\left(\overline{z-z_{0}}\right)^{t}\right]
\end{aligned}
$$

about the arbitrary point $\left(x_{0}, y_{0}\right)$. The representation (22) now follows.

If $n$ is $o d d$, then a similar discussion yields (23).

Sufficiency. First suppose that $n$ is even. If $f(x, y)$ has the representation (22), then $f(x, y)$ is both harmonic of order $k$ in $\mathcal{D}$ and satisfies $(18)$ in $\mathcal{D}$. Hence, as we have shown in the first part of the proof of this theorem, $f(x, y)$ has the representation (28) about the arbitrary, fixed point $\left(x_{0}, y_{0}\right)$. Now it is an easy matter to show that (7) holds for each $k$-tuple $\left\{p_{n}\left(x_{0}, y_{0} ; r_{k}, \phi\right)\right\}$ centered at $\left(x_{0}, y_{0}\right)$; we make use of (6) to do that. The conclusion of the lemma now follows.

If $n$ is an odd integer, $n \geqq 3$, we need but proceed as in the case for even $n$. This completes the proof.

CoROLlaRy. The conclusion of Theorem 1 is valid if (8) replaces (7) in the hypothesis.

It is interesting to note that for $k=1$, Theorem 1 reduces to a known characterization of harmonic polynomials $[1$, p. 236, p. 238]. If $k=2$, then Theorem 1 overlaps a result announced by Haskell [3]. Indeed, for $k=2$, (7) becomes

$$
\left|\begin{array}{lr}
L\left(f ; x, y ; r_{1}, \phi\right)-f(x, y) & r_{1}^{2} \\
L\left(f ; x, y ; r_{2}, \phi\right)-f(x, y) & r_{2}^{2}
\end{array}\right|=0 .
$$

A judicious integration of the left-hand member of (29), with respect to $r_{2}$, yields 


$$
\left|\begin{array}{cc}
L\left(f ; x, y ; r_{1}, \phi\right)-f(x, y) & r_{1}^{2} \\
A\left(f ; x, y ; r_{1}, \phi\right)-f(x, y) & r_{1}^{2} / 2
\end{array}\right|=0,
$$

and, consequently,

$$
f(x, y)=2 A\left(f ; x, y ; r_{1}, \phi\right)-L\left(f ; x, y ; r_{1}, \phi\right) .
$$

From Theorem 1 and (30) it follows that a necessary and sufficient condition that $f(x, y)$, continuous in $\mathcal{D}$, be a biharmonic polynomial of degree at most $n$ is that (30) hold for each $p_{n}\left(x, y ; r_{1}, \phi\right)$ lying in $D$. It is this result that is similar to a theorem obtained by Haskell.

If we allow $n \rightarrow \infty$ in (7), or (8), while $k$ is kept fixed, then we obtain characterizations of functions harmonic of order $k$ in $D$; these characterizations are contained in some results obtained by Poritsky [5], as well as by Choquet and Deny [2]. These latter should be compared with known results in the theory of functions harmonic of order $k, h \geqq 1$; see Nicolesco [4].

\section{BIBLIOGRAPHY}

1. E. F. Beckenbach and Maxwell Reade, Mean values and harmonic polynomials, Trans. Amer. Math. Soc. vol. 53 (1943) pp. 230-238.

2. G. Choquet and J. Deny, Sur quelques proprietes de moyennes ..., Bull. Soc. Math. France vol. 72 (1944) pp. 118-140.

3. R. N. Haskell, Mean values and biharmonic polynomials, Bull. Amer. Math. Soc. Abstract 52-9-294, p. 817.

4. Miron Nicolesco, Les fonctions polyharmoniques, Actualités Scientifiques et Industrielles, no. 331, Paris, 1936.

5. H. Poritsky, Generalizations of the Gauss law of the spherical mean, Trans. Amer. Math. Soc. vol. 43 (1938) pp. 199-225.

UNIVERSITY OF MichIGAN 